\title{
FDA одобрило два новых показания для применения препарата компании «MSD» КИТРУДА ${ }^{\circledR}$ (пембролизумаб)
}

Получено: 29.07.2019

Принято в печать: 02.08.2019

DOI: 10.32471/clinicaloncology.2663-466X.38.22853

Препарат КИтРУДА® (пембролизумаб) теперь одобрен для терапии первой линии у пациентов с метастатической или неоперабельной рецидивирующей плоскоклеточной карциномой головы и шеи в качестве монотерапии у больных, опухоли которых экспрессируют рецептор программируемой смерти клеток-1 (PD-L1; по комбинированной положительной шкале $\geq 1$ ), или в сочетании с платиной и фторурацилом независимо от уровня PD-L1-экспрессии.

Компания «MSD» сообщила, что Управление по контролю за пищевыми продуктами и лекарственными средствами США (Food and Drug Administration - FDA) одобрило препарат КИТРУДА ${ }^{\circledR}$ (пембролизумаб), лекарственное средство компании «MSD», воздействующее на рецептор программируемой смерти клеток-1 (PD-1), в качестве монотерапии у пациентов с опухолями, экспрессирующими PD-L1 (по комбинированной положительной шкале (combined positive score - CPS) $\geqslant 1)$ или в сочетании с платиной и фторурацилом - широко используемым режимом химиотерапии для терапии первой линии пациентов с метастатической или неоперабельной рецидивирующей плоскоклеточной карциномой головы и шеи. Одобрение основано на результатах ключевого клинического исследования III фазы KEYNOTE-048, в котором применение препарата КИТРУДА ${ }^{\circledR}$ обеспечило значительное улучшение общей выживаемости $(\mathrm{OB})$ по сравнению со схемой EXTREME (цетуксимаб с карбоплатином или цисплатином плюс фторурацил) - стандартной схемой лечения - как в качестве монотерапии у пациентов, опухоли которых экспрессировали PD-L1 (CPS $\geqslant 1$ ) (относительный риск $(\mathrm{OP})$ 0,78; 95\% доверительный интервал (ДИ) 0,64-0,96; $\mathrm{p}=0,0171$ ) и в сочетании с химиотерапией в общей популяции пациентов, принимавших участие в исследовании (ОР 0,$77 ; 95 \%$ ДИ $0,63-0,93 ; p=0,0067)$. После одобрения новых показаний к применению препарат КИТРУДА ${ }^{\circledR}$ стал первой терапией против PD-1, одобренной в качестве первой линии в виде монотерапии у пациентов, у которых опухоли экспрессируют PD-L1 (CPS $\geqslant 1)$, или в сочетании с химиотерапией независимо от уровня экспрессии PD-L1 для пациентов с метастатической или неоперабельной, рецидивирующей плоскоклеточной карциномой головы и шеи и первой анти-PD-1 терапией, продемонстрировавшей статистически достоверное улучшение ОВ у упомянутых пациентов.

«Это одобрение является впечатляющей новой вехой в терапии рака головы и шеи и может изменить методы лечения пациентов с этим изнурительным заболеванием, предлагая важные новые терапевтические возможности, - отметила доктор Барбара Бертнесс (Barbara Burtness), профессор медицины в Йельской медицинской школе (Yale School of Medicine) и содиректор Исследовательской программы развития терапии (Development Therapeutics Research Program) в Йельском онкологическом центре (Yale Cancer Center). - Метастатический или рецидивирующий рак головы и шеи является областью медицины, для которой характерно наличие существенных неудовлетворенных потребностей, поэтому необходимо располагать схемами иммунотерапии, доступными для пациентов в условиях первой линии терапии».

При применении препарата КИТРУДА ${ }^{\circledR}$ могут развиваться иммунно-опосредованные побочные реакции, в том числе тяжелые или фатальные, которые включают пневмонит, колит, гепатит, эндокринопатии, нефрит и по- чечную дисфункцию, тяжелые кожные реакции, отторжение трансплантата солидного органа и осложнения при аллогенной трансплантации гемопоэтических стволовых клеток. В зависимости от степени выраженности побочной реакции применение препарата КИТРУДА ${ }^{\circledR}$ следует прервать или прекратить, а при необходимости назначать кортикостероиды. КИТРУДА ${ }^{\circledR}$ также может вызвать серьезные или опасные для жизни реакции, связанные с инфузией. Учитывая механизм действия, КИТРУДА ${ }^{\circledR}$ может нанести в вред плоду при назначении беременной.

«Плоскоклеточная карцинома головы и шеи исторически была источником множества вызовов для врачей и пациентов, включая ограниченные варианты лечения, а также физические и функциональные проблемы, вызванные заболеванием и его лечением, - отметил доктор Джонатан Ченг (Jonathan Cheng), вице-президент по клиническим исследованиям «Merck Research Laboratories». - Это одобрение является важным достижением в лечении этого разрушающего заболевания. Результаты KEYNOTE-048, которые подтверждают данное утверждение, продемонстрировали, что монотерапия препаратом КИТРУДА ${ }^{\circledR}$ у пациентов, у которых опухоли экспрессировали PD-L1 (CPS $\geqslant 1)$, и КИТРУДА ${ }^{\circledR}$ в сочетании с химиотерапией вне зависимости от уровня экспрессии PD-L1 значительно увеличили выживаемость пациентов с метастатической или неоперабельной рецидивирующей плоскоклеточной карциномой головы и шеи в условиях первой линии терапии».

Препарат КИТРУДА ${ }^{\circledR}$ был первоначально одобрен для лечения пациентов с рецидивирующей или метастатической плоскоклеточной карциномой головы и шеи с прогрессированием заболевания во время или после платиносодержащей химиотерапии в 2016 г. в рамках ускоренного процесса одобрения FDA на основе объективных данных о частоте ответа из исследования Ib фазы KEYNOTE-012. В соответствии с условиями ускоренного процесса одобрения дальнейшее одобрение зависело от проверки и описания клинической пользы, что было продемонстрировано в KEYNOTE-048 и привело к тому, что FDA преобразовало ускоренное одобрение в полное (стандартное).

ДАННЫЕ, НА КОТОРЫХ ОСНОВАНО ОДОБРЕНИЕ

Одобрение новых показаний к применению препарата КИТРУДА ${ }^{\circledR}$ основано на данных предварительно запланированного промежуточного анализа клинического исследования III фазы KEYNOTE-048 - рандомизированного многоцентрового открытого исследования с активным контролем, в котором приняли участие 882 пациента с метастатической плоскоклеточной карциномой головы и шеи, которые ранее не получали системной терапии и которые считались неизлечимыми посредством местных методов терапии. Рандомизация была стратифицирована по уровню экспрессии PD-L1 опухолью (шкала пропорции опухоли (tumor proportion score - 
TPS) $\geqslant 50 \%$ или $<50 \%$ ) в соответствии с набором PD-L1 IHC $22 \mathrm{C} 3$ pharmDx kit, статус по наличию вируса папилломы человека (ВПЧ) в соответствии с р16 IHC (позитивный или негативный), и Общее состояние онкологического больного по шкале Восточной кооперативной онкологической группы (Eastern Cooperative Oncology Group - ECOG), статус производительности (performance status - PS) (0 против 1). Пациенты были рандомизированы 1:1:1 в одну из следующих групп терапии:

- пациенты, получавшие КИТРУДА ${ }^{\circledR} 200$ мг внутривенно каждые 3 нед;

- пациенты, получавшие КИТРУДА ${ }^{\circledR} 200$ мг внутривенно каждые 3 нед, карбоплатин AUC 5 мг/мл/мин внутривенно каждые 3 нед или цисплатин 100 мг/м² внутривенно каждые 3 нед и фторурацил 1000 мг/м²/сут в виде непрерывной внутривенной инфузии в течение 96 ч каждые 3 нед (максимум 6 циклов платины и фторурацила);

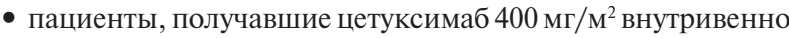
в качестве начальной дозы, затем 250 мг/м² внутривенно 1 раз в неделю, карбоплатин AUC 5 мг/мл/мин внутривенно каждые 3 нед или цисплатин 100 мг/м² внутривенно

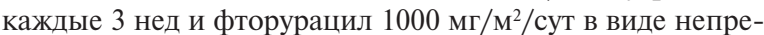
рывной внутривенной инфузии в течение 96 ч каждые 3 нед (максимум 6 циклов платины и фторурацила).

Характеристика популяции пациентов (882 пациента), принявших участие в исследовании: средний возраст 61 год (диапазон 20-94), 36\% в возрасте 65 лет и старше; 83\% мужчин; $73 \%$ европеоидной, $20 \%$ монголоидной и $2,4 \%$ негроид- ной расы; у 61\% отмечали PS ECOG 1; 79\% были бывшими или нынешними курильщиками. У $22 \%$ пациентов опухоли были ВПЧ-положительными; у 23\% отмечали количество опухолевых клеток (tumor proportion score - TPS) PD$\mathrm{L} 1 \geqslant 50 \%$; и у $95 \%$ была IV стадия заболевания (у 19\% - IVA стадия, у $6 \%$ - IVB стадия, и у $70 \%$ - IVC стадия). У $85 \%$ пациентов опухоли экспрессировали PD-L1 на уровне CPS $\geqslant 1$, a y $43 \%-$ CPS $\geqslant 20$.

Лечение препаратом КИТРУДА ${ }^{\circledR}$ продолжалось до тех пор, пока не было диагностировано прогрессирование заболевания по Критериям оценки объективного ответа при солидных опухолях v1.1 (Response Evaluation Criteria in Solid Tumors v1.1 - RECIST v1.1), определяемое исследователем, или была выявлена неприемлемая токсичность, или достигало 24 мес. Ретроспективная повторная классификация опухолевого статуса PD-L1 у пациентов в соответствии с CPS с использованием набора PD-L1 IHC 22C3 pharmDx kit проводилась с использованием образцов опухолей, которые использовали для рандомизации.

Основными показателями эффективности были ОВ и выживаемость без прогрессирования заболевания, которые оценивались с помощью слепой независимой централизованной оценки (blinded independent central review - BICR) в соответствии c RECIST v1.1 (модифицированным таким образом, чтобы отслеживать максимум 10 поражений и максимум 5 поражений на орган) и последовательно определялись в подгруппе пациентов с CPS $\geqslant 20$, в подгруппе пациентов с CPS $\geqslant 1$ и в общей популяции (табл. 1,2$)$.

Таблица 1. Показатели эффективности препарата КИТРУДАФ в качестве единственного агента в исследовании KEYNOTE-048 (CPS $\geqslant 1$ и CPS $\geqslant 20$ )

\begin{tabular}{|c|c|c|c|c|}
\hline \multirow[b]{2}{*}{ Конечная точка } & \multicolumn{2}{|c|}{ CPS $\geqslant 1$} & \multicolumn{2}{|c|}{ CPS $\geqslant 20$} \\
\hline & $\begin{array}{c}\text { КИТРУДА }^{\oplus} \\
200 \text { мг каждые } 3 \text { нед, } \\
\text { n=257 }\end{array}$ & $\begin{array}{c}\text { Цетуксимаб, платина, } \\
\text { фторурацил, } \\
\text { n=255 }\end{array}$ & $\begin{array}{c}\text { КИТРУДА }^{\oplus} \\
200 \text { мг каждые } 3 \text { нед, } \\
\text { n=133 }\end{array}$ & $\begin{array}{c}\text { Цетуксимаб, платина, } \\
\text { фторурацил, } \\
\text { n=122 }\end{array}$ \\
\hline \multicolumn{5}{|l|}{$\overline{O B}$} \\
\hline Количество событий (\%) & $177(69 \%)$ & $206(81 \%)$ & $82(62 \%)$ & $95(78 \%)$ \\
\hline Медиана, мес (95\% ди) & $12,3(10,8-14,9)$ & $10,3(9,0-11,5)$ & $14,9(11,6-21,5)$ & $10,7(8,8-12,8)$ \\
\hline $\mathrm{OP}^{\star}(95 \%$ ДИ) & \multicolumn{2}{|c|}{$0,78(0,64-0,96)$} & \multicolumn{2}{|c|}{$0,61(0,45-0,83)$} \\
\hline Значение $\mathrm{p}^{\dagger}$ & \multicolumn{2}{|c|}{0,0171} & \multirow{2}{*}{\multicolumn{2}{|c|}{0,0015}} \\
\hline \multicolumn{3}{|l|}{ ВБП } & & \\
\hline Количество событий (\%) & $225(88 \%)$ & $231(91 \%)$ & $113(85 \%)$ & $111(91 \%)$ \\
\hline Медиана, мес (95\% ди) & $3,2(2,2-3,4)$ & $5,0(4,8-5,8)$ & $3,4(3,2-3,8)$ & $5,0(4,8-6,2)$ \\
\hline ОР‡ (95\% ДИ) & \multicolumn{2}{|c|}{$1,15(0,95-1,38)$} & \multicolumn{2}{|c|}{$0,99(0,75-1,29)$} \\
\hline \multirow{2}{*}{\multicolumn{5}{|c|}{ Частота объективного ответа (ЧОО) }} \\
\hline & $19 \%(14,5-24,4)$ & $35 \%(29,1-41,1)$ & $23 \%(16,4-31,4)$ & $36 \%(27,6-45,3)$ \\
\hline Частота полного ответа, \% & 5 & 3 & 8 & 3 \\
\hline Частота частичного ответа, \% & 14 & 32 & 16 & 33 \\
\hline \multicolumn{5}{|l|}{ Длительность ответа } \\
\hline Медиана, мес (диапазон) & $20,9(1,5+, 34,8+)$ & $4,5(1,2+, 28,6+)$ & $20,9(2,7,34,8+)$ & $4,2(1,2+, 22,3+)$ \\
\hline
\end{tabular}

*На основании стратифицированной модели пропорциональных рисков Кокса.

${ }^{\dagger}$ НА основании стратификации по логранговому критерию.

ҒОтвет: лучший объективный ответ как подтвержденный полный ответ или частичный ответ.

Таблица 2. Показатели эффективности препарата КИТРУДА® в сочетании с платиной/фторурацилом в исследовании KEYNOTE-048

\begin{tabular}{|c|c|c|}
\hline Конечная точка & $\begin{array}{c}\text { КИТРУДАФ } 200 \text { мг каждые } 3 \text { нед, } \\
\text { платина, фторурацил, } \\
\text { n=281 }\end{array}$ & $\begin{array}{c}\text { Цетуксимаб, платина, } \\
\text { фторурацил, } \\
\text { n=278 }\end{array}$ \\
\hline \multicolumn{3}{|c|}{ 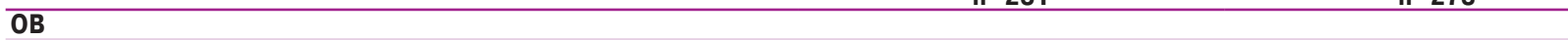 } \\
\hline Количество (\%) пациентов, у которых было зафиксировано событие (\%) & $197(70 \%)$ & $223(80 \%)$ \\
\hline Медиана, мес (95\% ДИ) & $13,0(10,9-14,7)$ & $10,7(9,3-11,7)$ \\
\hline $\mathrm{OP}^{\star}(95 \%$ ДИ) & \multicolumn{2}{|c|}{$0,77(0,63-0,93)$} \\
\hline Значение $\mathrm{p}^{\dagger}$ & \multicolumn{2}{|c|}{0,0067} \\
\hline \multicolumn{3}{|l|}{ ВБП } \\
\hline Количество событий (\%) & $244(87 \%)$ & $253(91 \%)$ \\
\hline Медиана, мес (95\% Ди) & $4,9(4,7,6,0)$ & $5,1(4,9,6,0)$ \\
\hline $\mathrm{OP}^{\ddagger}(Д И ~ 95 \%)$ & \multicolumn{2}{|c|}{$0,92(0,77-1,10)$} \\
\hline Значение $\mathrm{p}^{\dagger}$ & \multicolumn{2}{|c|}{0,3394} \\
\hline \multicolumn{3}{|l|}{400} \\
\hline $400^{\ddagger}(95 \%$ ДИ) & $36 \%(30,0-41,5)$ & $36 \%(30,7-42,3)$ \\
\hline Частота полного ответа, \% & 6 & 3 \\
\hline Частота частичного ответа, \% & 30 & 33 \\
\hline \multicolumn{3}{|l|}{ Длительность ответа } \\
\hline Медиана, мес (диапазон) & $6,7(1,6+, 30,4+)$ & $4,3(1,2+, 27,9+)$ \\
\hline
\end{tabular}

*На основании стратифицированной модели пропорциональных рисков Кокса.

${ }^{\dagger}$ На основании стратификации по логранговому критерию.

†Ответ: лучший объективный ответ как подтвержденный полный ответ или частичный ответ. 


\section{Актуально / Topical}

В ходе исследования КEYNOTE-048 профиль безопасности препарата КИТРУДА ${ }^{\circledR}$ как в качестве монотерапии, так и в сочетании с платиной (цисплатин или карбоплатин) и химиотерапией фторурацилом, был изучен у пациентов с ранее не леченной, рецидивирующей или метастатической плоскоклеточной карциномой головы и шеи. Средняя продолжительность применения препарата КИТРУДА ${ }^{\circledR}$ (200 мг каждые 3 нед) составила 3,5 мес (от 1 сут до 24,2 мес) в группе, получавшей монотерапию препаратом КИТРУДА ${ }^{\circledR}$, и 5,8 мес (от 3 сут до 24,2 мес) в группе, получавшей комбинацию препаратов.

Препарат КИТРУДА ${ }^{\circledR}$ был отменен из-за побочных реакций у $12 \%$ пациентов в группе, получавшей только КИТРУДА ${ }^{\circledR}$. Наиболее частыми побочными реакциями, приводящими к полному прекращению приема препарата КИТРУДА ${ }^{\circledR}$, были сепсис $(1,7 \%)$ и пневмония $(1,3 \%)$. Побочные реакции, приводящие к прерыванию терапии препаратом КИТРУДА имели место у $31 \%$ пациентов. Наиболее распространенными побочными реакциями, приводящими к прерыванию применения препарата КИТРУДА ${ }^{\circledR}(\geqslant 2 \%)$, были пневмония $(2,3 \%)$, пневмонит $(2,3 \%)$ и гипонатриемия $(2 \%)$. Наиболее распространенными побочными реакциями $(\geqslant 20 \%)$ при применении препарата КИТРУДА ${ }^{\circledR}$ в качестве единственного терапевтического агента были усталость (33\%), запор (20\%) и сыпь (20\%).

Препарат КИТРУДА ${ }^{\circledR}$ был отменен из-за побочных реакций у $16 \%$ пациентов в группе, получавшей комбинированную терапию. Наиболее частыми побочными реакциями, приводящими к полному прекрашению приема препарата КИТРУДА ${ }^{\circledR}$, были пневмония $(2,5 \%)$, пневмонит $(1,8 \%)$ и септический шок $(1,4 \%)$. Побочные реакции, приводящие к прерыванию терапии препаратом КИТРУДА ${ }^{\circledR}$, возникли у $45 \%$ пациентов. Наиболее распространенными побочными реакциями, требующими прекращения применения препарата КИТРУДА ${ }^{\circledR}(\geqslant 2 \%)$, были нейтропения $(14 \%)$, тромбоцитопения (10\%), анемия (6\%), пневмония $(4,7 \%)$ и фебрильная нейтропения $(2,9 \%)$. Наиболее распространенными побочными реакциями $(\geqslant 20 \%)$ при применении препарата КИТРУДА ${ }^{\circledR}$ в сочетании с платиной и фторурацилом были тошнота (51\%), усталость (49\%), запор (37\%), рвота (32\%), воспаление слизистых оболочек (31\%), диарея (29\%), снижение аппетита (29\%), стоматит $(26 \%)$ и кашель $(22 \%)$.

По материалам www.mrknewsroom.com 